\title{
Microorganisms under scrutiny
}

A microcystin ELISA, two blood culture systems, a confocal instrumentation reference, an automated agar sterilization and plate pouring system, and anaerobic workstations are among the products covered in this feature.

Cytosensor microphysiometer

From Molecular Devices

The 'Work Reference Guide' is the title of a 20-page brochure containing some 150 scientific references describing the uses of the microphysiometer

These applications include receptor activation and inactivation, signal transduction elucidation, agonist profiling, experimental in vitro toxicology, microbial metabolism, and growth factor, neurotrophic factor and cytokine responses. The reference guide also lists over 100 different cell types evaluated in the system, which is designed to detect responses triggered by a wide variety of receptor classes coupled to different second messengers. These receptor classes include G-protein linked receptors, ligandgated ion channels and tyrosine kinase receptors activated via growth factors, cytokines and neurotrophic factors.

Reader Enquiry No. 100

\section{Basic Z}

From Constant Systems

A continuous cell disrupter for bacterial, fungal, mammalian, plant or algal cells

With flow rates from $30 \mathrm{ml}$ to $80 \mathrm{ml} \mathrm{min}^{-1}$, the disrupter provides general disruption and release of subcellular particles, as well as the release of intracellular particles such as nucleic acids and proteins. The unit features a push-button start, an easy-to-clean design with no 'consumable' parts, a cooling facility, selective breakage, scale-up capability and no aerosol production. A 'one-shot' head adaptor is included for use with non-flowing samples and quantities from $1 \mathrm{ml}$ to $10 \mathrm{ml}$. Disruption is achieved through the use of high pressure, forcing

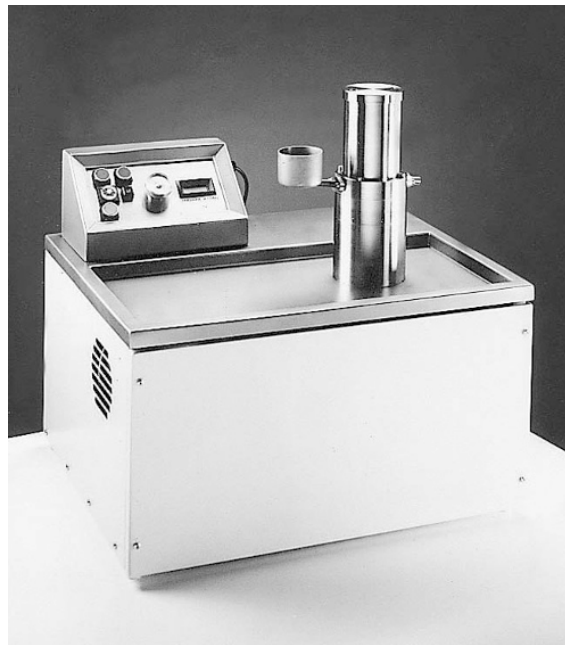

The Basic Z disrupter from Constant Systems. the sample through a small fixed orifice at high speed, without an increase in temperature. The pressure is adjustable up to $2.7 \times 10^{5} \mathrm{kPa}$ and is stated to be consistent and stable during the disruption cycle. Reader Enquiry No. 101

\section{Confocal instrumentation reference}

\section{From Bio-Rad}

A new 152-page bibliography cites a further 600 references that include the use of the company's confocal instrumentation

Research fields that have shown particularly strong recent growth include bacteriology/microbiology, pathology, embryology, neurobiology and biochemistry, especially drug discovery, says Bio-Rad. The reference includes full contact information for authors, as well as details of the fluorochromes and probes used in each paper. Available free of charge, the bibliography may prove to be a valuable reference source for research applying confocal microscopy. Other subject areas covered include genetics, materials science, experimental microscopy, parasitology and plant biology.

Reader Enquiry No. 102

\section{Microcystin ELISA kit}

\section{From Wako}

According to the manufacturer, the kit offers $a$ 50 to $1,600 \mathrm{pg} \mathrm{ml}^{-1}$ range of measurement, a sensitivity stated to be approximately $10^{3}$ times greater than with HPLC

Results are said to be obtained in as little as four hours, accounting for the presence of virtually all toxic microcystins. Microcystins, a group of cyclic heptapeptide hepatotoxins, are the most commonly reported toxins produced by the bloomforming cyanobacteria and are a primary cause of cyanobacterial poisoning. Besides their acute toxicity, it was recently reported that microcystin LR has tumour promoting activity in rats, as well as inhibiting protein phosphatase 1 and 2A. From these findings, microcystins have recently called attention to their role as possible environmental tumour promoters. This ELISA is said to offer convenient, solid-phase extraction-free quantification. In the normal assay, formate incubation time is stated to be $18-26 \mathrm{~h}$ with a sensitivity of $60 \mathrm{pg} \mathrm{ml}^{-1}$, whereas, the short assay format takes as little as $3 \mathrm{~h}$ with a sensitivity of $100 \mathrm{pg} \mathrm{ml}^{-1}$. The doubleassay method accommodates 43 samples and the single-assay method 91 samples. Reader Enquiry No. 103

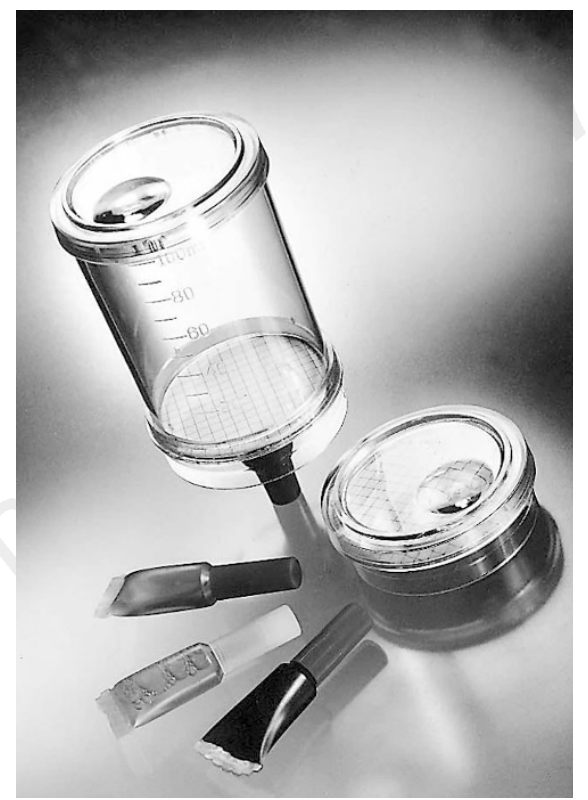

Sartorius' microbiological monitor comes with presterilized membrane, Petri dish and media.

\section{Microbiological monitor}

From Sartorius

A 100-ml microbiological monitor that is designed to lower costs

Sterile and disposable, the monitor has a 47-mm diameter cellulose nitrate membrane with a large bottom port that gives faster flow rates and decreased testing times. It has been designed for the easy detection of microorganisms in beverages and water, but is suitable for many other applications. Available as a complete presterilized package (membrane, Petri dish and media), it is said to do away with time-intensive steps involved in setting up steel funnels, flame sterilizing and preparing media. Another feature of the new unit is the integral magnifying disc on the cover, which enables the user to examine any colonies that have appeared on the membrane surface by simply rotating the disc. The standard monitor package contains 50 sterile disposable monitor units, 50 coloured bottom port plugs and a large funnel adapter and a small funnel adapter.

Reader Enquiry No. 104

\section{Densimat}

From bioMerieux

A system designed for density of suspensions destined for use with manual or automated identification and susceptibility test strips

The use of this new densitometer should help improve the utility and reliability of 


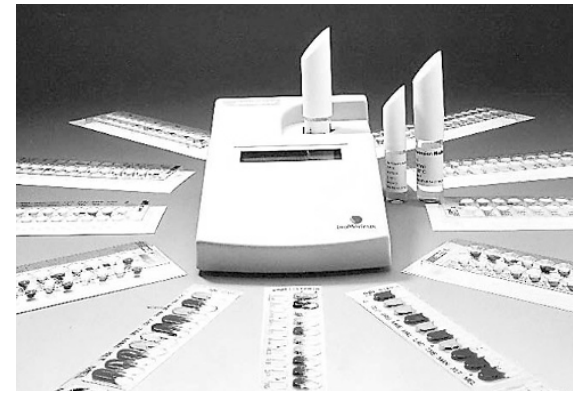

Densimat from bioMerieux is for use with

identification and susceptibility test strips.

API, ID32, rapid ID32, ATB, and rapid ATB strips. Easy-to-handle and compact, the Densimat weighs only $0.5 \mathrm{~kg}$ and runs on its own integral lithium batteries. Operation is said to be simple — as soon as an ampoule is inserted, the instrument reads the microbial density. The results are then displayed on the LCD panel within the range of 0.5-7.5 McFarland units. A new 44-page, full-colour catalogue describing the entire bioMerieux range of products for industrial and environmental microbiology is now available.

Reader Enquiry No. 105

\section{Wedia and preparation}

\section{Sterile Blood}

From TCS Microbiology

Sterile animal blood in PVC bags for cost-

effective bacteriological media preparation

With the needs of the larger scale blood user in mind, the company has designed a system that offers a reduction in the contamination risk associated with the use of bottles. The company states that it provides packed-cell volumes with minimal risk, quality-control sampling and reduced wastage. Litre volumes and upward are available and are said to carry the reassurance of adherence to a BS EN ISO 9002 accredited quality assurance system incorporating rigorous visual and chemical QC procedures.

\section{Reader Enquiry No. 106}

\section{BBL product brochure}

From Becton Dickinson Microbiology

Systems

The company offers support in the standardization of prepared media and consumer cost maintenance

The support offered includes helping the clinical laboratory achieve standardization of optimal media, for example, adopting the fewest number of ideal products necessary to achieve the high-quality testing results. A full-colour brochure is is available to help the laboratory manager choose from the many plated media formulations that are available.

Reader Enquiry No. 107

\section{Rediprep}

From Mast Diagnostics

An extensive range of high-quality prepared media in plate, bottle and tube formats

All media are prepared under BS EN ISO9002 registered conditions and are said to be compliant with laboratory accreditation needs. The company offers microbiology laboratories a flexible service for the supply of poured plates and bottled media. Flexible ordering systems are in place to deal with changing needs, or standing orders can be put into place to ensure continuity of supply. The range includes standard media for general use and specialized media. Other media not listed can be manufactured to order, but are subject to a minimum order requirement. Although specifically designed for UK laboratories, the company will discuss export options with interested customers. The range includes Ready Poured Plates: single media, Bi-plates and contact plates, as well as specialized plates, irradiated media (plates and bottles) and bottledprepared media.

Reader Enquiry No. 108

\section{AS-10 automatic media preparation}

From Brunswick Scientific

An automated agar sterilization and plate pouring system

A quarter turn in and a quarter turn out allows for speedy removal and insertion of the dispense valve and thermowell, as well as a larger, more convenient addition port. Headplate components are smaller so that they are more accessible and easier to handle and the headplate itself is light and easily rotated to give unobstructed access, according to the manufacturer. Media preparation times are said to be faster as well, producing 2-10 1 of ready-to-pour, high-quality agar within just one hour. The AS10's stirring mechanism helps to prevent caramelization to ensure highquality agar. It couples directly with automatic pourer/stackers, inkjet batch identification systems and programmable dispensing pumps to provide a complete, automated service to the busy media preparation laboratory.

Reader Enquiry No. 109

\section{Signal and Isolator \\ From Oxoid \\ Two blood culture systems that provide complementary diagnostic approaches for general purpose and specialist applications}

Signal is a one-bottle system containing a specially formulated broth medium that allows the culture of aerobes, anaerobes and microaerophilic organisms for general bacteraemia and fungaemia screening. Isolator is a more specialized detection and isolation system based on direct draw tubes, and is said to be suited for use in cases where fungaemia, low-level bacteraemia or infection by fastidious microorganisms is suspected. In the Signal blood culture system, blood is inoculated into individual bottles, each of which carries a growth indicator device. Gas production from growth activity generates positive pressure in the bottle, displacing some of the blood/broth mixture into the device. Samples can be removed directly from the device for subculturing purposes, without having to enter the bottle itself. According to the manufacturer, the clear detection of microbial growth provided by Signal facilitates the rapid isolation, identification and antimicrobial susceptibility testing of microorganisms from blood culture. Isolator is said to be a rapid and highly sensitive tube-based blood culture system that detects and isolates microorganisms in a single process. Tubes are available in two sizes: an adult version for use with 10-ml blood samples, and a paediatric version that can be used to culture samples ranging from $0.5 \mathrm{ml}$ to $1.5 \mathrm{ml}$ in volume. In both cases, tubes are used for phlebotomy, as well as subsequent sample processing. The manufacturer states that the use of lysis centrifugation and direct plating technology means that colonies are available an average $12-14 \mathrm{~h}$ sooner than would be possible with conventional broth-based or biphasic methods.

\section{Reader Enquiry No. 110}

\section{Wall Guide}

From Sigma

This free wall guide highlights microbial media and components

This easy-to-use, alphabetical reference includes descriptions and other pertinent information on over 85 different microbial media and associated products used in biotechnology. The convenient wall reference lists over three-dozen general and specialized microbial media products, such as brain-heart fusion agar, Luria broth base, and thioglycollate medium. Also featured is a diverse selection of media components, including bacteriological agars, protein hydrolysates and yeast extracts, as well as components for specialized applications.

Reader Enquiry No. 111

\section{WASP plater}

From Don Whitley Scientific

This automatic spiral plater (WASP) has minimal set up and the option to select and 'lock' user-defined parameters - variable or uniform deposition, fill mode and plate size

A single keystroke initiates the complete cycle, including loading of sample, plating out and cleaning. The microprocessor- 


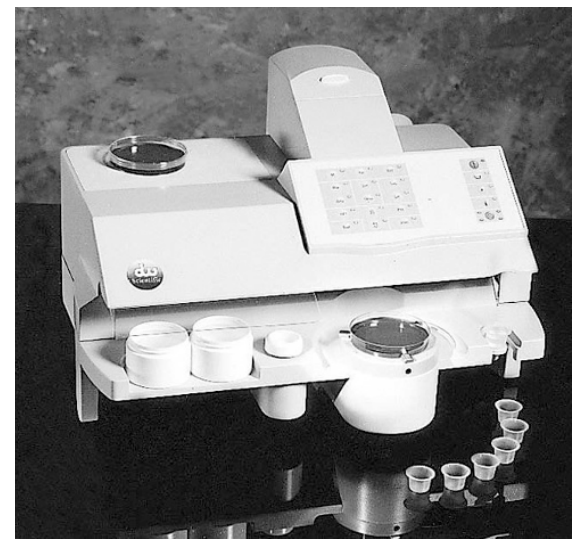

The WASP automatic spiral plater from Don Whitley has a microprocessor-controlled syringe.

controlled sample syringe has a capacity of $250 \mu \mathrm{l}$, which allows flexibility of total volume dispensed and a consequent increase in the machine's sensitivity range. Microprocessor control has the added benefits that multiple plates can be inoculated without the need to refill the syringe. A choice of uniform or variable deposition modes is available for all possible sample volumes. WASP will inoculate a plate at the fastest speed possible for any particular volume, carefully optimizing the centrifugal force to ensure that sample is not pushed to the edge of the plate. After inoculation is complete, the cleaning cycle is automatically initiated, drawing upon fresh sterile water and five per cent hypochlorite solution to ensure effective sterilization of the tip. Users can be removing one plate and preparing the next while cleaning is under way. All parameters are continually monitored by the unit's CPU.

Reader Enquiry No. 112

\section{Incubators and cabinets}

Shel Lab 2323/TC

From Borolabs

Designed to be a low-cost $\mathrm{CO}_{2}$ incubator, incorporating a warm air-jacket design that provides temperature uniformity usually only found in application-specific models

The new models have had their exterior redesigned to be easier to use - all control parameters, including calibration, can now be set from the front-mounted touch-pad panel. A parallel printer or computer connection port has also been incorporated to allow users to export data from the incubator to any IBM-compatible printer. The port can also be used for remote monitoring of temperature and $\mathrm{CO}_{2}$ alarm conditions. Borolabs also offers a free $\mathrm{CO}_{2}$ changeover unit that will automatically start using a new $\mathrm{CO}_{2}$ cylinder when one is running low. Such features as an integral microprocessor that ensures precise temperature and $\mathrm{CO}_{2}$ control regardless of how the chamber is loaded have been retained. Humidification is achieved through evaporation, and rela- tive humidity is displayed with an accuracy of 1.0 per cent. A $25-\mathrm{mm}$ access port accommodates through-wall operation of electrical equipment within the chamber and a tempered glass viewing door allows the scientist to view samples without loss of temperature, $\mathrm{CO}_{2}$ or humidity. The other main design feature of the incubator is its triple-wall construction. An extra large waterjacket surrounds the incubator chamber and over this a secondary layer of insulation has been added to the side walls. This design offers temperature uniformity throughout the incubator and helps to eliminate the possibility of cold spots within the chamber which may result in condensation - a primary source of contamination. Reader Enquiry No. 113

\section{BH12R and BH12D safety cabinets}

\section{From Labcaire}

Two new class II microbiological safety cabinets designed to meet the airflow and structural requirements of BS 5726 (1992)

With high levels of user protection, the clean sheet design also has low noise levels and ergonomic simplicity. Oversized filters and high-torque fans are said to ensure low noise and high performance, while low-mounted, fully electronic touch controls ensure ease of operation for all users. According to the manufacturer, both units provide sample protection by bathing work surfaces with $0.12-\mu \mathrm{m}$ filtered air. A one-piece, crevicefree work surface is said to be easy to clean and helps avoid contamination. Electronics and filters are front mounted to simplify servicing. Moreover, there is optimized internal lighting and a generous 1,200 -mm stainless steel work surface to provide a comfortable working environment. Large HEPA filters are sealed under negative pressure to eliminate leaks. The recirculatory

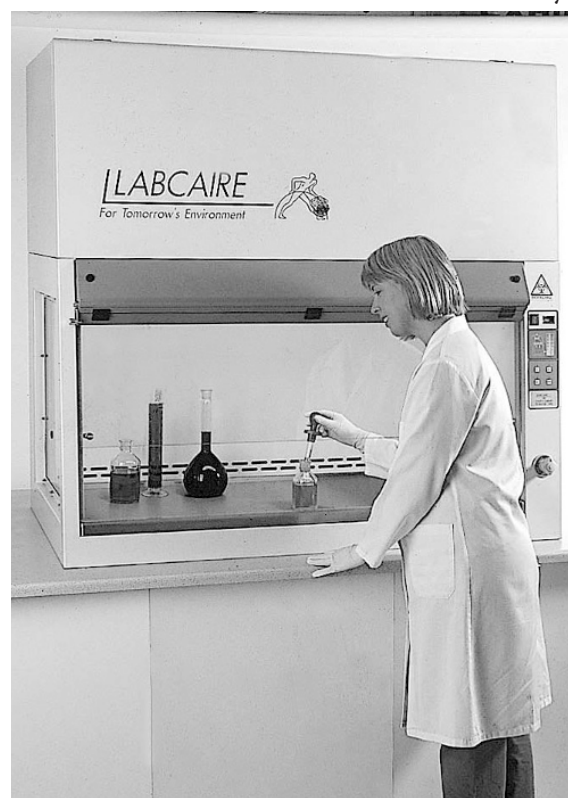

Class II microbiology cabinets from Labcaire.
BH12R and ducted $\mathrm{BH} 12 \mathrm{D}$ units share the dimensions $1,500 \times 1,340 \times 780 \mathrm{~mm}(\mathrm{H} \times$ $\mathrm{W} \times \mathrm{D})$. Optional stands and other accessories, such as ultraviolet lights and formalin vapourizer units, are available to order.

Reader Enquiry No. 114

Conceptanaerobic workstations

From Baker Scientific

There are three models within this range,

which includes the Concept Plus, Concept 300 and Bugbox workstations

The largest model in the range is Concept Plus, which requires only 1,600 $\mathrm{mm}$ of bench space and has been designed to address the needs of the larger laboratory where sample throughput and incubator capacity are paramount. This unit offers appropriate growth conditions for anaerobes, has a large incubated work area, a storage facility for up to 500 plates with an 'economy' purge cycle for small batch transfer. The Concept 300 has a footprint measuring just $900 \times 650 \times 750$ $\mathrm{mm}$ and can accommodate up to 400 plates. A novel tube interlock facilitates transfer of up to $11 \times 90-\mathrm{mm}$ Petri dishes in $15 \mathrm{~s}$, whereas a single-plate entry system allows individual dishes to be transferred directly to the cabinet. Standard features of these two models include dual-gas operation, barehand access, automatic humidity control and nitrogen change-over with microprocessor monitoring and alarm systems. Completing the range is the Bugbox, a small and sophisticated unit, says the company, which includes all the standard features of the larger workstations and is intended for smaller research projects and clinical laboratories. It accommodates up to 180 plates, making the costly use of anaerobic jars redundant, and offers precise temperature and humidity controls. An optional feature of all Concept units is the connection of a data logging system and chart recorder for 24-h monitoring.

\section{Reader Enquiry No. 115}

\section{Odds and ends}

Surespin 630

From Sorvall

A swinging-bucket rotor that has been designed to be an easy-to-use, top-loading rotor with greater sample security

The rotor should help to eliminate sampleloading errors in ultracentrifuge operations. Its top-access design makes attachment pins clearly visible, allowing easy verification that each bucket is properly seated and thus enhancing sample protection. The rotor has been built for speeds up to 30,000 r.p.m. In addition, it has cavities that guide buckets easily into place, such that they can be loaded and unloaded without having to remove the rotor body from the centrifuge. Furthermore, bayonet-style covers require no special tools to seal. The Surespin rotor 


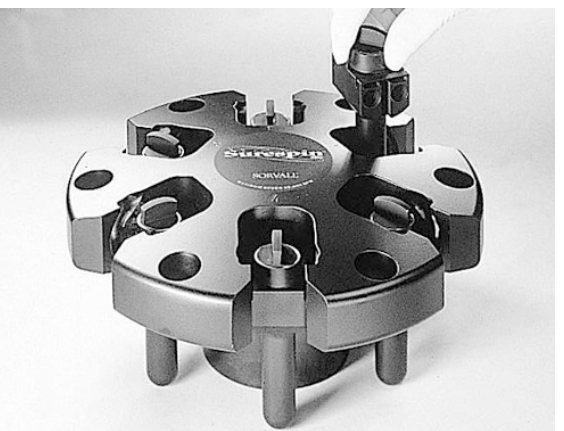

Sorvall's Surespin 630 swinging-bucket rotor.

is designed for compatibility with other competitive ultracentrifuges.

Reader Enquiry No. 116

\section{Centricon Plus $=20$ and $=80$}

From Millipore

Part of the company's family of centrifugal filtration devices

These devices are easy-to-use and may replace stirred-cell systems, dialysis and other methods that require time to set-up, maintenance procedures or additional processing steps. The devices incorporate the high-flow Biomax membrane for fast processing or the high-recovery YM membrane. They also offer the invert spin method of concentrates recovery, allowing for improved sample recovery. Centricon Plus-20 concentrates samples of $20 \mathrm{ml}$ down to $150 \mathrm{pl}$ in as little as $10 \mathrm{~min}$; whereas, Centricon Plus-80 allows for concentration of $80 \mathrm{ml}$ down to $300 \mathrm{pl}$ in as little as $30 \mathrm{~min}$. These devices fit any $50 \mathrm{ml}$ and $250 \mathrm{ml}$ swinging bucket rotors.

Reader Enquiry No. 117

\section{Master/Amp kit}

From Epicentre Technologies

$P C R$ kits and enzymes incorporate a new technology that is designed to improve PCR

According to the manufacturer, amplification consistency and yields are increased, and poor, unreliable or failed amplification reactions are eliminated with the company's kits and enzymes. This includes templates that cannot be amplified using conventional PCR because of GC-rich regions or extensive secondary structure. Other MasterAmp products include PCR optimization kits,

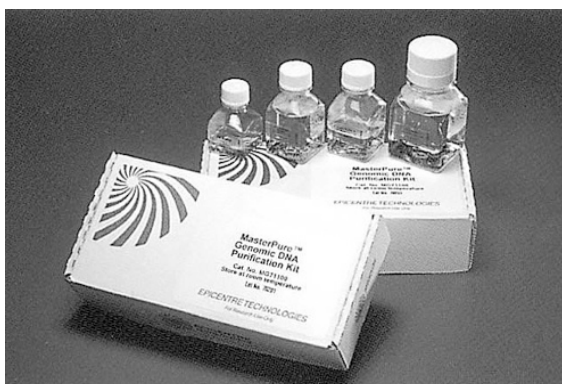

MasterAmp PCR kits from Epicentre.
PCR core kits, and PCR-qualified Taq, Tfl, Tth and AmpliTherm DNA polymerases. Reader Enquiry No. 118

\section{Ultrapure RapidGel solutions}

From Amersham

A wide range of premixed liquid acrylamide to simplify DNA sequencing

These solutions have been formulated to allow DNA sequencing gels to be poured in minutes and still retain high levels of reproducibility. Stated to be suitable for both radioactive and fluorescent sequencing electrophoresis, the solutions are stable, premixed and available in a range of convenient percentages in either TBE $(4,4.75,5,6$ and 8 per cent) or GTG (4, 6 and 8 per cent) formulations; a 40 per cent stock concentrate is also available. The RapidGel-XL high-resolution matrix is for use in DNA sequencing and is stated to yield up to 40 per cent more readable sequence per gel. It is available either as ready-to-use solutions (4, 6 and 8 per cent) or in a 40 per cent stock concentrate, which can be used for the preparation of custom percentages.

Reader Enquiry No. 119

\section{FP500 Econo}

From Ultra-Lum

A new modular gel system for economical, efficient photography, documentation and analysis of gels

This low-cost system uses a high-resolution CCD camera mounted on the UltraLum low-profile darkroom. The darkroom is designed to fit on any UltraLum transilluminators and can be equipped with optional ultraviolet lighting, incident or epi-illumination. Incremental, modular additions can be made with a 256 gray-scale thermal printer, for example, providing photography at less than ten cents a picture, or a frame grabber that will plug into either an IBM- or Macintosh-formatted computer for complete documentation and analysis. The PHD 2000 can be installed providing documentation capability with image enhancement. The KS3000 option can convert this basic setup into a full visualization, documentation and analysis system.

Reader Enquiry No. 120

\section{Multichannel Gel Loading Syringes} From Hamilton

Syringes that allow users to transfer up to 12 samples at a time from microwell plates to even the thinnest electrophoresis gels

There are three available needle sizes, including a new $0.2-\mathrm{mm}$ design for deeper loading between plates and into wells. Adjustable volume stops allow faster and more accurate reloading and improve reproducibility. The syringes allow quick

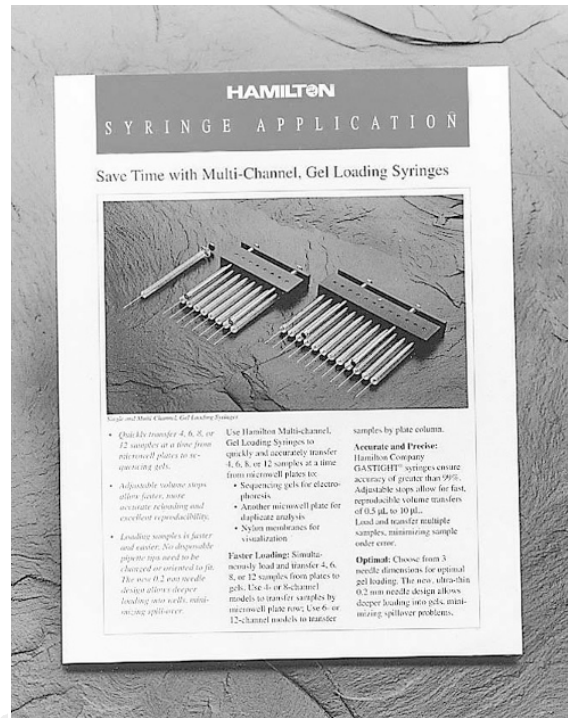

Hamilton's multichannel syringes allow the efficient transfer of 4, 6, 8 and 12 samples.

transfer of 4, 6, 8 and 12 samples at a time from microwell plates to sequencing gels. Adjustable volume stops allow faster, more accurate reloading and excellent reproducibility. No disposable pipette tips need to be changed or oriented to fit. Models for 4- or 8-channel transfer by microwell plate row and 6- or 12-channel transfer plate column are available. Gas-tight syringes are said to ensure accuracy of greater than 99 per cent, and adjustable stops allow for fast, reproducible volume transfers of $0.5-10 \mu \mathrm{l}$

Reader Enquiry No. 121

These notes are compiled by Brendan Horton from information provided by the manufacturers. For more details, fill in the reader service card bound inside the journal.

ADVERTISEMENT

CUSTOM HYBRIDOMA DEVELOPMENT

- Ascites Production

- Purification - Conjugation

- Polyclonal Antibodies Also Available

1-800-481-9737

e-mail: antibodies@htibio.com http://www.htibio.com
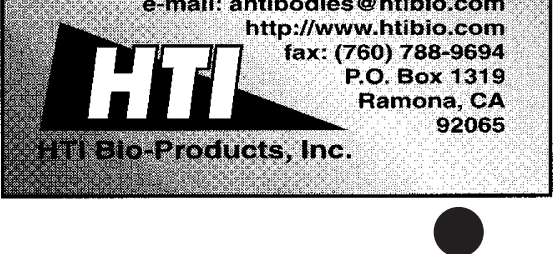